\title{
RESEARCH
}

Open Access

\section{Is comprehensiveness critical? Comparing short and long format cognitive assessments in preclinical Alzheimer disease}

\author{
Jason Hassenstab ${ }^{1,2^{*}}$, Jessica Nicosia', Megan LaRose', Andrew J. Aschenbrenner ${ }^{1}$, Brian A. Gordon²,3,
} Tammie L. S. Benzinger ${ }^{3}$, Chengjie Xiong ${ }^{1,4}$ and John C. Morris ${ }^{1}$

\begin{abstract}
Background: Comprehensive testing of cognitive functioning is standard practice in studies of Alzheimer disease (AD). Short-form tests like the Montreal Cognitive Assessment (MoCA) use a "sampling" of measures, administering key items in a shortened format to efficiently assess cognition while reducing time requirements, participant burden, and administrative costs. We compared the MoCA to a commonly used long-form cognitive battery in predicting $A D$ symptom onset and sensitivity to $A D$ neuroimaging biomarkers.

Methods: Survival, area under the receiver operating characteristic (ROC) curve (AUC), and multiple regression analyses compared the MoCA and long-form measures in predicting time to symptom onset in cognitively normal older adults ( $n=6230$ ) from the National Alzheimer's Coordinating Center (NACC) cohort who had, on average, 2.3 \pm 1.2 annual assessments. Multiple regression models in a separate sample $(n=416)$ from the Charles F. and Joanne Knight Alzheimer Disease Research Center (Knight ADRC) compared the sensitivity of the MoCA and longform measures to neuroimaging biomarkers including amyloid PET, tau PET, and cortical thickness.

Results: Hazard ratios suggested that both the MoCA and the long-form measures are similarly and modestly efficacious in predicting symptomatic conversion, although model comparison analyses indicated that the longform measures slightly outperformed the MoCA (HRs > 1.57). AUC analyses indicated no difference between the measures in predicting conversion (DeLong's test, $Z=1.48, p=0.13$ ). Sensitivity to AD neuroimaging biomarkers was similar for the two measures though there were only modest associations with tau PET ( $r s=-0.13, p s<0.02$ ) and cortical thickness in cognitively normal participants ( $r s=0.15-0.16, p s<0.007)$.
\end{abstract}

\footnotetext{
* Correspondence: hassenstabj@wustl.edu

${ }^{1}$ Charles F. and Joanne Knight Alzheimer Disease Research Center,

Department of Neurology, Washington University School of Medicine, St. Louis, MO, USA

2Department of Psychological \& Brain Sciences, Washington University in St. Louis, St. Louis, MO, USA

Full list of author information is available at the end of the article
}

(c) The Author(s). 2021 Open Access This article is licensed under a Creative Commons Attribution 4.0 International License, which permits use, sharing, adaptation, distribution and reproduction in any medium or format, as long as you give appropriate credit to the original author(s) and the source, provide a link to the Creative Commons licence, and indicate if changes were made. The images or other third party material in this article are included in the article's Creative Commons licence, unless indicated otherwise in a credit line to the material. If material is not included in the article's Creative Commons licence and your intended use is not permitted by statutory regulation or exceeds the permitted use, you will need to obtain permission directly from the copyright holder. To view a copy of this licence, visit http://creativecommons.org/licenses/by/4.0/. The Creative Commons Public Domain Dedication waiver (http://creativecommons.org/publicdomain/zero/1.0/) applies to the data made available in this article, unless otherwise stated in a credit line to the data. 
Conclusions: Both test formats showed weak associations with symptom onset, AUC analyses indicated low diagnostic accuracy, and biomarker correlations were modest in cognitively normal participants. Alternative assessment approaches are needed to improve how clinicians and researchers monitor cognitive changes and disease progression prior to symptom onset.

Keywords: Alzheimer disease, Cognitive decline, Cognitive assessment

\section{Introduction}

Detecting changes in cognition in the earliest stages of Alzheimer disease (AD) in longitudinal studies requires that assessment measures meet several criteria. Assessments must be psychometrically valid and reliable and test relevant domains of cognition such as episodic memory, executive function, attentional control, processing speed, language, and orientation. For both overall scores and domain-specific scores, these measures should have good retest reliability and inter-rater reliability. Of particular importance, especially for studies that enroll large asymptomatic cohorts, is that the tests included are sensitive enough to detect subtle changes in cognition that characterize the preclinical asymptomatic stages of $\mathrm{AD}[1,2]$. Attempting to maximize sensitivity, AD cohort studies and clinical trials typically involve extensive cognitive testing sessions including dozens of individual measures. The comprehensiveness of a cognitive evaluation is often valued as a method to provide more nuanced and sensitive insights into cognitive changes commonly associated with AD pathology [3, 4]. However, this comprehensiveness comes at a cost as lengthier evaluations can be problematic for multiple reasons. First, longer assessments increase participant burden and require significant personnel time for test administration, scoring, quality control, and data entry ${ }^{1}$. Second, day-to-day variability in stress and mood can significantly impact participants' attention and fatigue, factors which have been shown to particularly influence older adults' cognitive performance [5]. Finally, the tests which tap into the domains of cognition that decline in the earliest stages of $\mathrm{AD}$, including episodic memory, attention, and working memory, are perceived as most taxing and effortful $[6,7]$ and often have suboptimal reliability [8].

Short-form cognitive assessment tools, sometimes referred to as cognitive screening measures, have been used for decades in clinical studies to provide a snapshot of overall cognitive and functional abilities and require a fraction of the time it takes to complete their long-form counterparts. The brevity and simplicity of these assessments make them popular tools among researchers and

\footnotetext{
${ }^{1}$ Additionally, in light of the COVID-19 pandemic, it is worth noting that the length of long-form cognitive assessments may also make them less adaptable to remote testing.
}

clinicians when assessing cognitive decline. The most common short-form measure in the AD literature is the Mini-Mental State Examination (MMSE) [9]. The MMSE requires approximately $10 \mathrm{~min}$ to administer and has high sensitivity and specificity for detecting symptomatic $\mathrm{AD}[10]$. However, at milder stages of dementia, this test has exhibited poor sensitivity [11]. Specifically, due to ceiling effects, individuals with preclinical AD or early symptomatic $\mathrm{AD}$ (e.g., mild cognitive impairment [MCI] due to AD) are more likely to score within the "normal" range [12-14]. Beyond these validity and reliability issues, the MMSE is subject to copyright restrictions and carries fees for its use [15, 16], making it costly for large-scale studies and restricts research to investigators with sufficient resources.

For these reasons, the MMSE was not included in the National Alzheimer's Coordinating Center (NACC) Uniform Data Set (UDS) in its third iteration (UDS 3) [17, 18] in lieu of the Montreal Cognitive Assessment (MoCA) [19], a short-form cognitive assessment similar to the MMSE with several advantages. The MoCA requires approximately $10-15 \mathrm{~min}$ to administer and tests seven domains of cognition: memory, visuospatial function, naming, attention, language, orientation, and abstraction. The most unique feature of the MoCA is that, apart from simple assessments of orientation and abstraction like those included in the MMSE, it uses items and test concepts drawn from classic long-form neuropsychological test batteries. The MoCA includes condensed versions, or a "sampling," of more comprehensive measures including the trail making test, letter fluency, confrontation naming, digit span, and verbal list learning and recall. Like the MMSE, it is highly sensitive to dementia, but unlike the MMSE, it is more sensitive to early symptomatic AD [14, 20]; has shown sensitivity to $\mathrm{AD}$ biomarkers [21]; and is available at no cost for non-profit use, although its publisher has recently begun requiring training and certification fees (https://www.mocatest.org/training-certification/).

The goal of the current study was to determine how the short-form MoCA measure compares to standard long-form neuropsychological tests in terms of predicting the onset of symptomatic dementia in the NACC cohort. The NACC cohort is a large, well-characterized sample of older adults enrolled in ongoing studies of aging and dementia at $\sim 30$ Alzheimer's Disease 
Research Centers across the USA. Additional analyses were done using participants enrolled in studies at the Charles F. and Joanne Knight Alzheimer Disease Research Center (Knight ADRC) to determine the sensitivity of the short-form and long-form measures to $\mathrm{AD}$ neuroimaging biomarkers, including amyloid positron emission tomography (PET), tau PET, and magnetic resonance imaging (MRI) structural measures. We hypothesized that, in comparison with the MoCA, the long-form cognitive measures from the UDS 3 would show superior specificity and sensitivity in predicting conversion to symptomatic $\mathrm{AD}$ and would be more sensitive to $\mathrm{AD}$ neuroimaging biomarkers.

\section{Study 1}

Methods

Participants

The NACC UDS 3 is a standardized evaluation consisting of clinical and cognitive measures administered to participants enrolled in ongoing studies of aging and dementia at $\sim 30$ centers funded by the National Institute on Aging (NIA) Alzheimers Disease Research Center (ADRC) program. Written informed consent is obtained at the individual ADRCs and approved by individual Institutional Review Boards (IRBs). We included participant visits submitted to NACC by the ADRCs from March of 2015 to August 2019. This time period reflects when the MoCA and other UDS 3 measures were introduced into the ADRCs and submitted to NACC. Because we were interested in determining the utility of the MoCA as compared to the long-form UDS 3 cognitive battery in predicting disease progression from cognitive normality to onset of symptomatic disease, we included only participants who were cognitively normal at their first visit when MoCA was introduced into the ADRCs. Participants were required to have a Clinical Dementia Rating ${ }^{\mathrm{mm}}\left(\mathrm{CDR}^{\mathrm{m}}\right)$ [22] of 0 at their first visit and have at least one follow-up visit.

\section{Clinical and cognitive measures}

Clinical status was determined with the CDR which uses a 5-point scale to characterize six domains of cognitive and functional performance that are applicable to $\mathrm{AD}$ and other dementias [22]. The domains include memory, orientation, judgment and problem solving, community affairs, home and hobbies, and personal care. CDR scores are determined through semi-structured interviews with the participant and a reliable informant such as a family member or friend. A CDR score of 0 indicates cognitive normality, $0.5=$ very mild dementia, $1=$ mild dementia, $2=$ moderate dementia, and $3=$ severe dementia.

The UDS 3 cognitive battery includes measures of episodic memory (Craft Story 21, Benson Complex Figure Recall), language (the Multilingual Naming Test
(MINT)), visuospatial functioning (Benson Complex Figure Copy), immediate attention (Trails A, Number Span Forward), working memory (Number Span Backwards), and executive functioning (Trails B; see Weintraub et al., 2018 for a detailed description and associated references for individual measures). Each test was standardized using the mean and standard deviation from the first visit for individuals who remained CDR 0 (i.e., nonconverters) to form $Z$-scores. $Z$-scores from each test were then averaged together to form domain scores (i.e., memory, visual, attention, language) which were then averaged together to form a simple global composite score. Because the MoCA total score is commonly used in the diagnosis of cognitive impairment [23], our analyses focused on comparing the MoCA total score with the UDS 3 global composite score.

\section{Statistical analyses}

Time to symptomatic conversion was operationalized as the time, in years, from participants' initial study time point to the time point in which they were first determined to have a non-zero CDR score (or, in the case of individuals who never converted to a non-zero CDR, their final time point in the study). Individuals who died during follow-up were not included in the analyses (i.e., 148 non-converters and 31 converters). Two cut points, one at $-1 \mathrm{SD}$ and one at $-1.5 \mathrm{SDs}$, were used for both the MoCA (scores of 23 and 21, respectively) and the UDS 3 global composite score. Similar cut points have been used in the literature to indicate the presence or absence of cognitive impairment [24-28]. Log-rank tests were used to compare the Kaplan-Meier survival curves for high and low groups for each measure (i.e., the MoCA and the UDS 3 global composite). Adjusted hazard ratios (HRs) and their 95\% confidence intervals were estimated using a Cox proportional hazards model with covariates including baseline age, self-reported gender, years of education, and presence of one or more apolipoprotein $\varepsilon 4$ (APOE4) alleles with the survival package version 3.2 in the $R$ statistical computing environment [29].

To obtain measures of the two tests' diagnostic discrimination abilities [30], the scales were subjected to an area under the curve (AUC) analysis of receiver operating characteristics (ROC) curves using the $p R O C$ package version 1.17 in $R$ [30]. This analysis focused on examining which score provided better diagnostic accuracy in predicting conversion status using baseline data from individuals who were CDR 0 at their initial visit. The ROC analysis results were interpreted following the diagnostic accuracy guidelines from Swets (1996) such that an AUC $<0.70$ indicates low diagnostic accuracy, an AUC in the range of $0.70-0.90$ indicates moderate diagnostic accuracy, and an AUC $\geq 0.90$ indicates high 
diagnostic accuracy [30]. DeLong's test for correlated ROC curves was used to test whether the two areas under the curve were significantly different from one another.

Finally, multiple regression model comparison analyses were used to directly compare the efficacy of the baseline, continuous MoCA, and UDS 3 global composite scores in predicting the time to symptomatic conversion. Because the MoCA and UDS 3 global composite were correlated with one another, $r=0.70$, $p<0.001$, a single multiple regression model including both variables would be subject to potential multicollinearity issues. Therefore, non-nested models were used, and model fit was evaluated based on the $R^{2}$, Akaike information criterion (AIC), Bayesian information criterion (BIC), and deviance values. For all models, males served as the reference group for the gender variable, and APOE negative served as the reference groups for the APOE variable.

\section{Results}

Demographics

Six thousand two hundred thirty cognitively normal older adults aged $72.9 \pm 10.4$ years from the NACC cohort were followed for an average of $2.3 \pm 1.2$ annual assessments (range $=1-5$ assessments). As expected, converters had more assessments than non-converters, $t(746.92)=12.90, p<0.001, d=0.55$. Participants that enroll as cognitively normal that are observed for longer periods are more likely to convert due to advancing age (see Table 1 for additional demographic information).

\section{Survival model results}

Survival analyses were conducted using outcomes from the short-form MoCA and the long-form UDS 3 global composite to predict the time to symptomatic onset (defined as a change from CDR 0 to CDR $>0$ ). Percentages of high- and low-scoring converters and non-converters for each cut point are presented in Table 2. Outcomes were contrasted on HR magnitude and their confidence intervals. For reference, higher HRs are interpreted as representing a better prediction of future conversion and narrower confidence intervals suggest less variability in prediction. Primary analyses contrasted individuals who scored above vs. below the $-1 \mathrm{SD}$ and -1.5 SDs cut points on the MoCA and UDS 3 global composite. HRs for symptomatic conversion by the cut point criteria described above, adjusted for demographic covariates, are

Table 1 Study 1 demographic data

\begin{tabular}{|c|c|c|c|}
\hline Converter & No, $N=5622^{1}$ & Yes, $N=608^{1}$ & $p$-value ${ }^{2}$ \\
\hline Age & $73(7)$ & $76(8)$ & $<0.001$ \\
\hline Gender & & & 0.002 \\
\hline Female & $3709(66 \%)$ & $362(60 \%)$ & \\
\hline Male & $1913(34 \%)$ & $246(40 \%)$ & \\
\hline Education (years) & $16.24(2.81)$ & $16.08(3.09)$ & 0.24 \\
\hline APOE status & & & 0.4 \\
\hline Neg. & $2608(70 \%)$ & $351(68 \%)$ & \\
\hline Pos. & $1134(30 \%)$ & $167(32 \%)$ & \\
\hline Race & & & 0.21 \\
\hline White & $4499(80 \%)$ & $480(79 \%)$ & \\
\hline Black or African American & $853(15 \%)$ & $107(18 \%)$ & \\
\hline Asian & $148(2.6 \%)$ & $10(1.6 \%)$ & \\
\hline American Indian or Alaska Native & $40(0.7 \%)$ & $7(1.2 \%)$ & \\
\hline Native Hawaiian or other Pacific Islander & $6(0.1 \%)$ & $0(0 \%)$ & \\
\hline Others & $32(0.6 \%)$ & $2(0.3 \%)$ & \\
\hline Unknown & $44(0.8 \%)$ & $2(0.3 \%)$ & \\
\hline Number of visits & $2.25(1.2)$ & $3.21(0.94)$ & $<0.001$ \\
\hline MoCA & $26.1(2.9)$ & $24.6(3.3)$ & $<0.001$ \\
\hline UDS 3 global composite & $0.05(0.57)$ & $-0.25(0.59)$ & $<0.001$ \\
\hline
\end{tabular}

${ }^{1}$ Mean (SD); $n$ (\%)

${ }^{2}$ Welch two-sample $t$-test; Pearson's chi-squared test 
Table 2 Study 1 cut point Ns

\begin{tabular}{|c|c|c|}
\hline Converter & No, $N=5622^{1}$ & Yes, $N=608^{1}$ \\
\hline \multicolumn{3}{|c|}{ MoCA - 1 SD cut point } \\
\hline High & $4710(84 \%)$ & $416(68 \%)$ \\
\hline Low & $912(16 \%)$ & $192(32 \%)$ \\
\hline \multicolumn{3}{|c|}{ Global composite - 1 SD cut point } \\
\hline High & $5381(96 \%)$ & $543(89 \%)$ \\
\hline Low & $241(4.3 \%)$ & $65(11 \%)$ \\
\hline \multicolumn{3}{|c|}{ MoCA - 1.5 SD cut point } \\
\hline High & $5204(93 \%)$ & $505(83 \%)$ \\
\hline Low & $418(7.4 \%)$ & $103(17 \%)$ \\
\hline \multicolumn{3}{|c|}{ Global composite -1.5 SD cut point } \\
\hline High & $5537(98 \%)$ & $591(97 \%)$ \\
\hline Low & $85(1.5 \%)$ & $17(2.8 \%)$ \\
\hline
\end{tabular}

presented in Table 3. For all models, the adjusted cumulative incidence of time to symptomatic conversion was increased in the individuals who scored below the cut point after controlling for demographic covariates as compared to individuals who scored above the cut point, HRs $>1.57, p s<0.001$. Comparison of HRs indicated that the UDS 3 global composite was slightly better at predicting symptomatic conversion than the MoCA with the -1 SD cut point. However, the opposite was true (MoCA HR > UDS 3 global composite HR) with the $1.5 \mathrm{SD}$ cut point (see Table 3). Overall, these results ultimately suggest that both the MoCA and the UDS 3 global composite show relatively equivalent HRs and are thus similarly, yet modestly, efficacious in predicting symptomatic conversion. Kaplan-Meier curves for the MoCA and UDS 3 global composite at the two different cut points are presented in Fig. 1.
ROC results

ROC analyses in the NACC sample indicated an area under the curve (AUC) estimate for the MoCA of 0.64 and an AUC estimate for the UDS 3 global composite of 0.66. DeLong's test for two correlated ROC curves was non-significant, $Z=1.48, p=0.13$, indicating that these measures demonstrate similarly low diagnostic accuracy in predicting conversion to symptomatic $\mathrm{AD}[31]$.

\section{Model comparison results}

The multiple regression models predicting the time to symptomatic conversion indicated that both the continuous MoCA and UDS 3 global composite scores significantly predicted time to symptomatic conversion after covarying out effects of age, self-reported gender, years of education, and APOE4 status. The results of the model comparison analyses suggested that the model including the UDS 3 global composite score outperformed the model including the MoCA score in predicting time to symptomatic conversion. This was indicated by the larger $R^{2}$ and smaller AIC, BIC, and deviance values (see Table 4).

\section{Study 2 \\ Methods \\ Participants}

Participants were community-residing volunteers enrolled in longitudinal studies of memory and aging at the Knight ADRC at Washington University in St. Louis. We were interested in determining the associations between the MoCA and long-form measures with $\mathrm{AD}$ neuroimaging biomarkers, so we selected participants based on the availability of at least 1 amyloid PET scan, 1 tau PET scan, and a volumetric MRI that were collected

Table 3 HRs for time to symptomatic conversion by task score adjusted for covariates

\begin{tabular}{|c|c|c|c|c|c|c|c|c|c|c|c|c|}
\hline \multirow[t]{2}{*}{ Predictor } & \multicolumn{3}{|c|}{ MoCA - 1 SD cut point } & \multicolumn{3}{|c|}{ MoCA - 1.5 SD cut point } & \multicolumn{3}{|c|}{ Composite - 1 SD cut point } & \multicolumn{3}{|c|}{ Composite -1.5 SD cut point } \\
\hline & HR & $95 \% \mathrm{Cl}$ & $p$ & HR & $95 \% \mathrm{Cl}$ & $p$ & HR & $95 \% \mathrm{Cl}$ & $p$ & HR & $95 \% \mathrm{Cl}$ & $p$ \\
\hline Age & 1.02 & $1.01,1.04$ & $<0.001$ & 1.03 & $1.01,1.04$ & $<0.001$ & 1.03 & $1.02,1.04$ & $<0.001$ & 1.03 & $1.02,1.04$ & $<0.001$ \\
\hline Gender & 0.76 & $0.64,0.91$ & 0.003 & 0.72 & $0.60,0.86$ & $<0.001$ & 0.74 & $0.62,0.89$ & 0.001 & 0.74 & $0.62,0.88$ & $<0.001$ \\
\hline Education & 1.00 & $0.97,1.03$ & $>0.9$ & 1.00 & $0.97,1.04$ & 0.90 & 0.99 & $0.96,1.02$ & 0.40 & 0.97 & $0.94,1.00$ & 0.035 \\
\hline APOE & 1.22 & $1.01,1.47$ & 0.04 & 1.20 & $0.99,1.44$ & 0.06 & 1.23 & $1.02,1.49$ & 0.03 & 1.22 & $1.01,1.47$ & 0.038 \\
\hline \multicolumn{13}{|l|}{ MoCA } \\
\hline High & - & - & - & - & - & - & & & & & & \\
\hline Low & 2.99 & $2.45,3.65$ & $<0.001$ & 3.87 & $3.00,4.99$ & $<0.001$ & & & & & & \\
\hline \multicolumn{13}{|c|}{ Global composite } \\
\hline High & & & & & & & - & - & - & - & - & \\
\hline Low & & & & & & & 4.06 & $2.95,5.58$ & $<0.001$ & 2.69 & $1.57,4.61$ & $<0.001$ \\
\hline
\end{tabular}

$H R$ hazard ratio, $\mathrm{Cl}$ confidence interval 

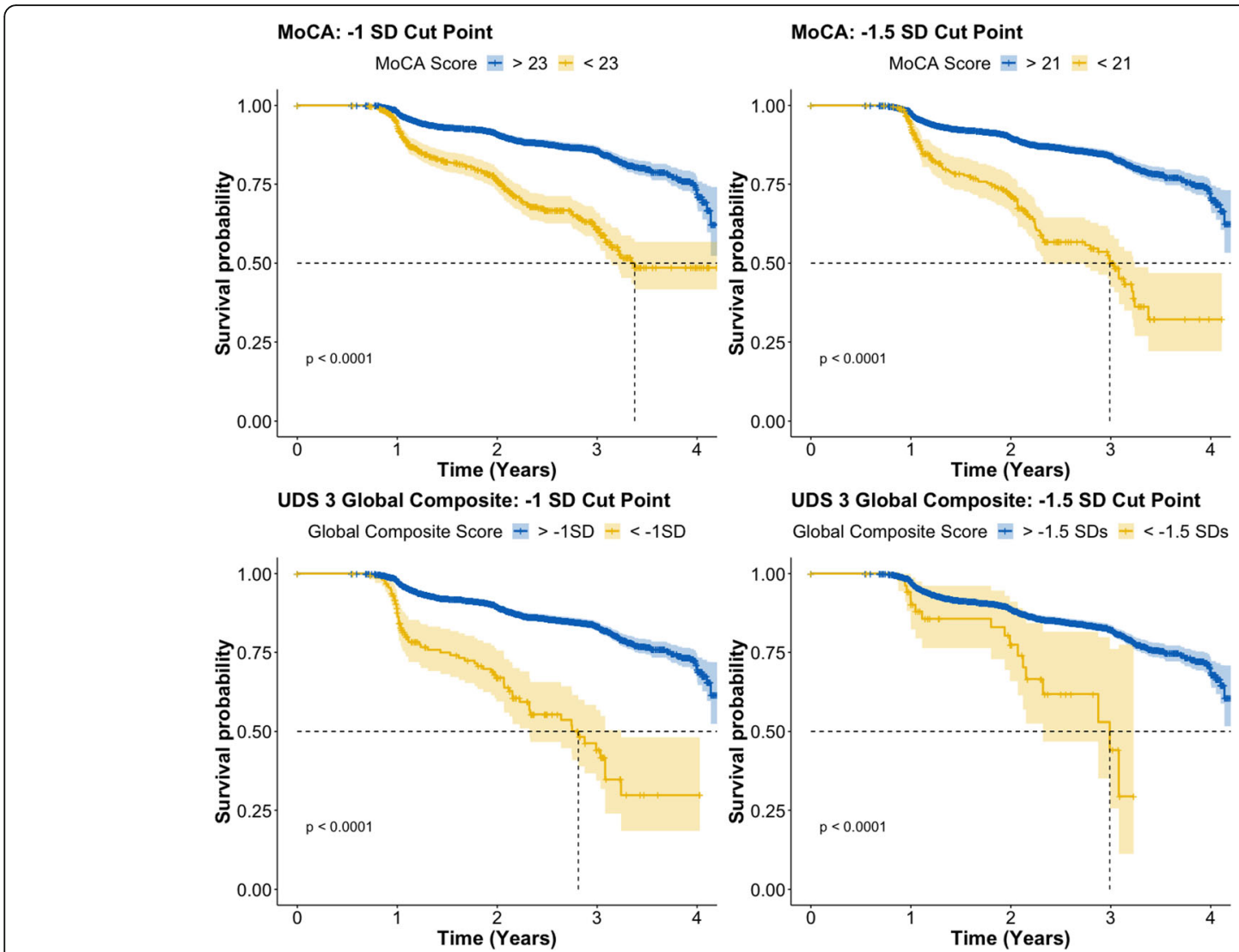

Fig. 1 Survival curves for the MoCA and UDS 3 global composite for the two different cut points ( 1 SD and - 1.5 SDs)

within 1 year of each other. We also sought to determine the sensitivity of the MoCA and long-form measures in tracking disease progression from the asymptomatic to symptomatic stages; thus, data were analyzed in two groups: participants who were CDR 0 and participants who were $C D R>0$. All participants signed a standard informed consent document, and all procedures were approved by the Institutional Review Board at Washington University in St. Louis.

\section{Clinical and cognitive measures}

Measures included the CDR (as described in study 1), MoCA, and UDS 3 (as described in study 1). The UDS 3

Table 4 Study 1 model comparisons

\begin{tabular}{|c|c|c|c|c|c|c|}
\hline \multirow[t]{2}{*}{ Predictor } & \multicolumn{3}{|c|}{ MoCA model } & \multicolumn{3}{|c|}{ Global composite model } \\
\hline & Beta & $95 \% \mathrm{Cl}$ & $p$ & Beta & $95 \% \mathrm{Cl}$ & $p$ \\
\hline Age & 0.00 & $-0.01,0.01$ & 0.60 & 0.00 & $-0.01,0.01$ & 0.80 \\
\hline Gender & 0.13 & $-0.02,0.28$ & 0.09 & 0.11 & $-0.04,0.26$ & 0.14 \\
\hline Education & -0.03 & $-0.06,0.00$ & 0.05 & -0.03 & $-0.06,-0.01$ & 0.01 \\
\hline APOE & 0.06 & $-0.10,0.22$ & 0.40 & 0.06 & $-0.09,0.22$ & 0.40 \\
\hline Standardized MoCA & 0.11 & $0.04,0.18$ & 0.003 & & & \\
\hline Global composite & & & & 0.30 & $0.17,0.44$ & $<0.001$ \\
\hline$R^{2}$ & 0.028 & & & 0.05 & & \\
\hline AlC & 1292 & & & 1280 & & \\
\hline BIC & 1322 & & & 1310 & & \\
\hline Deviance & 362 & & & 353 & & \\
\hline
\end{tabular}


global composite was calculated in the same way as described in study 1.

\section{Neuroimaging}

Amyloid PET imaging was performed with either florbetapir (18F-AV-45) or Pittsburgh Compound B (PiB) and was acquired on a Biograph mMR (Siemens Medical Solutions, Malvern, PA). All florbetapir PET scans include data 50- to 70-min post-injection, and all PiB PET scans include data 30- to 60-min post-injection. All data were converted to standardized uptake value ratios (SUVRs) with the cerebellar cortex used as a reference region before then being converted to the Centiloid scale [32, 33]. PET data were processed with an in-house pipeline using regions of interest derived from FreeSurfer (https://github.com/ysu001/PUP) [34]. This approach corrects for the spillover signal from adjacent regions of interest and non-brain tissue on the basis of the scanner point spread function and the relative distance between regions. This partial volume correction approach accounts not only for spillover from different areas in the brain but also for spillover from the non-brain regions into the brain. Amyloid deposition was quantified with the average across the left and right lateral orbitofrontal, medial orbitofrontal, rostral middle frontal, superior frontal, superior temporal, middle temporal, and precuneus regions. Tau PET imaging used the tracer 18F-AV1451 (flortaucipir) and was acquired on a Biograph 40 PET/CT scanner (Siemens Medical Solutions). Data from the 80- to 100-min post-injection window were converted to SUVRs using a cerebellar cortex reference and partial volume corrected. Deposition was summarized with the average of the bilateral entorhinal cortex, amygdala, inferior temporal lobe, and lateral occipital cortex [35]. MRI data were acquired on a Siemens Biograph mMR or Trio 3T scanner. T1-weighted images were acquired with a magnetization-prepared rapid acquisition gradient echo sequence acquired in the sagittal orientation with a repetition time of $2300 \mathrm{~ms}$, an echo time of $2.95 \mathrm{~ms}$, a flip angle of $9^{\circ}, 176$ slices, an in-plane resolution of $240 \times 256$, and a slice thickness of $1.2 \mathrm{~mm}$. Images underwent volumetric segmentation with FreeSurfer 5.3 (freesurfer.net) to identify the regions of interest for further analysis [36, 37]. Cortical thickness values were obtained for each hemisphere for a limited number of regions of interest (ROIs) reflecting the brain atrophy patterns in AD [38]. Cortical thickness was calculated as the shortest distance between the cortical gray/white boundary to the gray/CSF boundary [39].

\section{Statistical analyses}

The relationships between the AD neuroimaging biomarkers (at baseline) and the MoCA/UDS3 global composite in CDR 0 and CDR 0.5 individuals were examined using multiple regression models in the $\mathrm{R}$ statistical computing environment. As in study 1, due to the high degree of correlation between the MoCA and UDS 3 global composite scores, non-nested model comparison metrics (i.e., $R^{2}, \mathrm{AIC}, \mathrm{BIC}$, and deviance) were used in lieu of a single, nested multiple regression model to avoid potential confounding multicollinearity issues. Separate models were run predicting each biomarker and separately for each subset of the data (i.e., CDR $0 \mathrm{~s}$, CDR $0.5 \mathrm{~s}$ ) using demographic predictor variables as the covariates and either the MoCA or the UDS 3 global composite as the key independent variable. For all models, males served as the reference group for the gender variable, and APOE negative served as the reference group for the APOE variable.

\section{Results}

\section{Demographics}

Participants included 416 mostly older adults aged 45 to 92 years old enrolled in ongoing studies at the Knight ADRC (see Table 5 for the complete demographic information) and who had completed at least one relevant biomarker scan and a clinical assessment. Most participants $(n=365)$ were cognitively normal with a CDR of 0.

\section{Regression results}

As shown in Table 6, analyses of CDR 0 participants indicated small but significant relationships between both short- and long-form scores (i.e., the MoCA and UDS 3 global composite) and tau PET and cortical thickness ( $p$ s 0.007-0.03). Model comparison results indicated that the MoCA and UDS 3 global composite were relatively similarly sensitive in predicting biomarker values as indicated by the $R^{2}$, AIC, BIC, and deviance values (see Table 6). As expected, in the CDR 0.5 subsample, there were stronger relationships between the MoCA and UDS 3 global composite and all of the neuroimaging biomarkers, including amyloid PET, as compared to the CDR 0 sample (see Table 7). Model comparison analyses indicated that the MoCA was a better predictor of biomarker levels as compared to the UDS 3 global composite in the CDR 0.5. Scatterplots showing the relationships between the MoCA and UDS 3 global composite score and the three neuroimaging biomarkers, with separate regression lines for CDR 0 and $0.5 \mathrm{~s}$, are displayed in Fig. 2. As shown in the scatterplots, and as made evident by the regression models, both the MoCA and UDS 3 global composite were essentially equivalent in their associations with the neuroimaging $\mathrm{AD}$ biomarkers.

\section{General discussion}

The present study compared the efficacy of a popular short-form cognitive measure (MoCA) against a global 
Table 5 Study 2 demographic data

\begin{tabular}{|c|c|c|c|}
\hline CDR & $0, N=365^{1}$ & $0.5, N=51^{1}$ & $p^{2}$ \\
\hline Age & $71(6)$ & $74(6)$ & $<0.001$ \\
\hline Gender & & & 0.12 \\
\hline Female & $211(58 \%)$ & $23(45 \%)$ & \\
\hline Male & $154(42 \%)$ & $28(55 \%)$ & \\
\hline Education (years) & $16(2)$ & $16(3)$ & 0.076 \\
\hline APOE & & & 0.001 \\
\hline Neg. & $245(67 \%)$ & $22(43 \%)$ & \\
\hline Pos. & $119(33 \%)$ & $29(57 \%)$ & \\
\hline Race & & & 0.83 \\
\hline White & $320(88 \%)$ & 45 (88\%) & \\
\hline Black or African American & $41(11 \%)$ & $5(9.8 \%)$ & \\
\hline Others (American Indian, Alaska Native, Native Hawaiian, Pacific Islander, or Asian) & $4(1.1 \%)$ & $1(2.0 \%)$ & \\
\hline MoCA & $26(2)$ & $22(4)$ & $<0.001$ \\
\hline UDS 3 global composite & $0.22(0.58)$ & $-0.62(0.73)$ & $<0.001$ \\
\hline Amyloid PET (Centiloid) & $17(24)$ & $59(40)$ & $<0.001$ \\
\hline Tau PET SUVR & $1.22(0.19)$ & $1.64(0.56)$ & $<0.001$ \\
\hline AD ROI cortical thickness (mm) & $2.82(0.14)$ & $2.64(0.21)$ & $<0.001$ \\
\hline
\end{tabular}

${ }^{1}$ Mean (SD); $n(\%)$

${ }^{2}$ Welch two-sample $t$-test; Pearson's chi-squared test

composite score created from the more comprehensive long-form UDS3 cognitive battery in predicting AD progression. $\mathrm{AD}$ progression was operationalized as the time to the onset of initial symptoms. Additionally, we compared the short- and long-form measures on their sensitivity to $\mathrm{AD}$ neuroimaging biomarkers. Using data from the NACC, we compared the survival analyses, ROC curves, and multiple regression models using the MoCA total score and a global cognitive composite to investigate whether one measure outperformed the other. In a sample of data from the Knight ADRC, which included several neuroimaging biomarkers in addition to both the MoCA and UDS 3 cognitive battery, we further investigated the predictive power of each score in predicting biomarker levels in participants who were cognitively normal (CDR 0 ) and in those with very mild dementia (CDR 0.5).

Our first hypothesis, that the long-form measure would outperform the MoCA in predicting disease progression, was supported, although both measures produced only modest associations. We compared commonly used standard deviation (SD) cut points, at $-1.0 \mathrm{SD}$ and $-1.5 \mathrm{SD}$, for both measures. Similar cut points have been used in the literature to indicate the presence or absence of cognitive impairment [24-28]. HRs from the survival analyses indicated that both the MoCA and UDS 3 global composite were relatively equivalent in their sensitivity to symptomatic conversion, though ROC analyses indicated that neither reached the threshold for acceptable diagnostic accuracy. Model comparison analyses in the NACC sample indicated that the UDS 3 global composite outperformed the MoCA by only a slim margin.

It appears that longer, more comprehensive cognitive assessments may afford a marginal benefit over shorter cognitive screening measures like the MoCA when used to predict the time to symptomatic disease onset. In contrast, when stricter cut points were used (i.e., - 1.5 SDs), the MoCA outperformed the long-form composite score. These findings should be interpreted with caution, however, because there are far fewer cases at this low-performance threshold for the long-form global composite than the MoCA (see Table 2 and confidence interval magnitude in Fig. 1), which was expected given that the sample was selected based on a baseline CDR of 0 . These data also corroborate existing studies which find that cutoff scores, which purportedly identify individuals at the greatest risk for cognitive decline and symptom onset [2], have unacceptably poor predictive power, even when coupled with $\mathrm{AD}$ biomarker information [40].

We also hypothesized that the long-form global composite would prove superior to the MoCA in associations with $\mathrm{AD}$ neuroimaging biomarkers including amyloid PET, tau PET, and an AD-specific ROI cortical thickness measure. However, analyses in the 


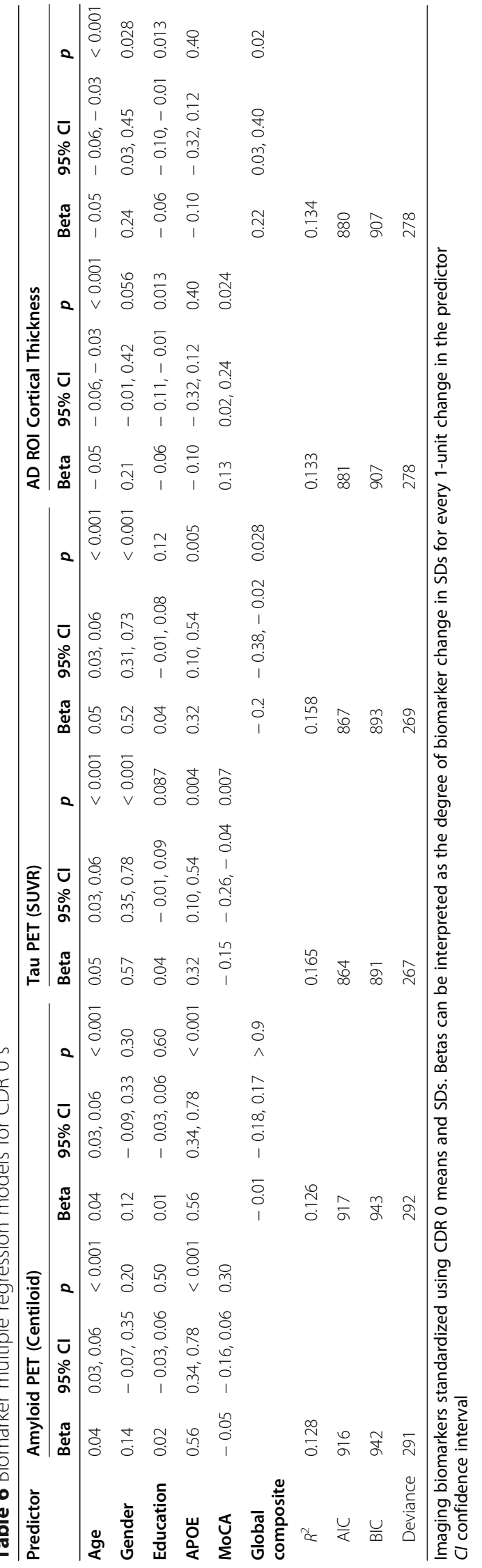




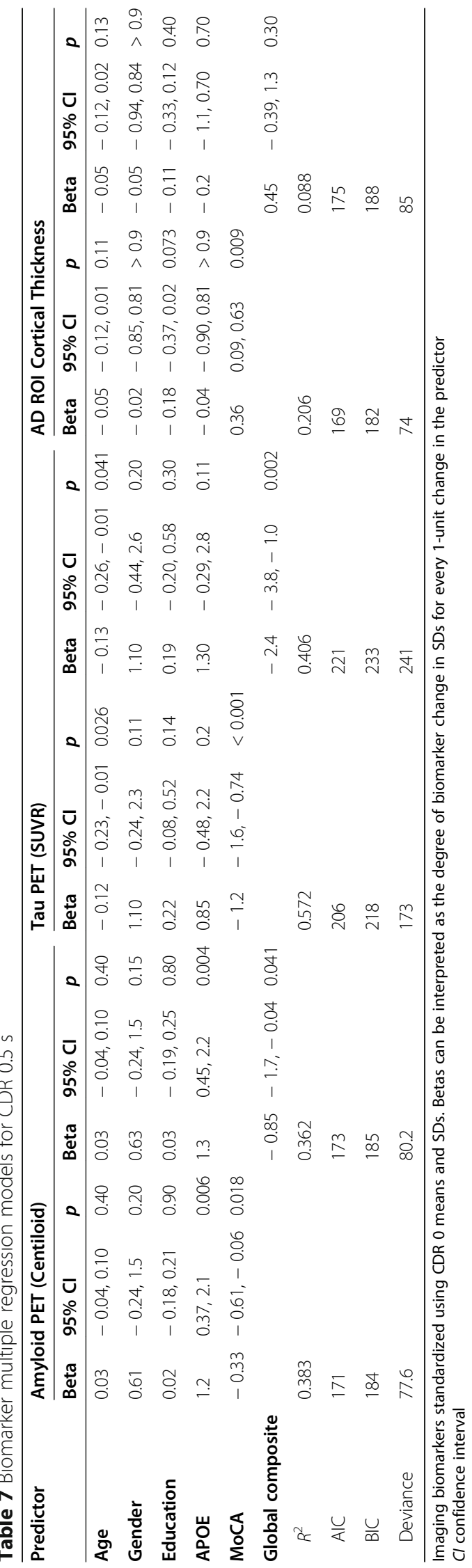



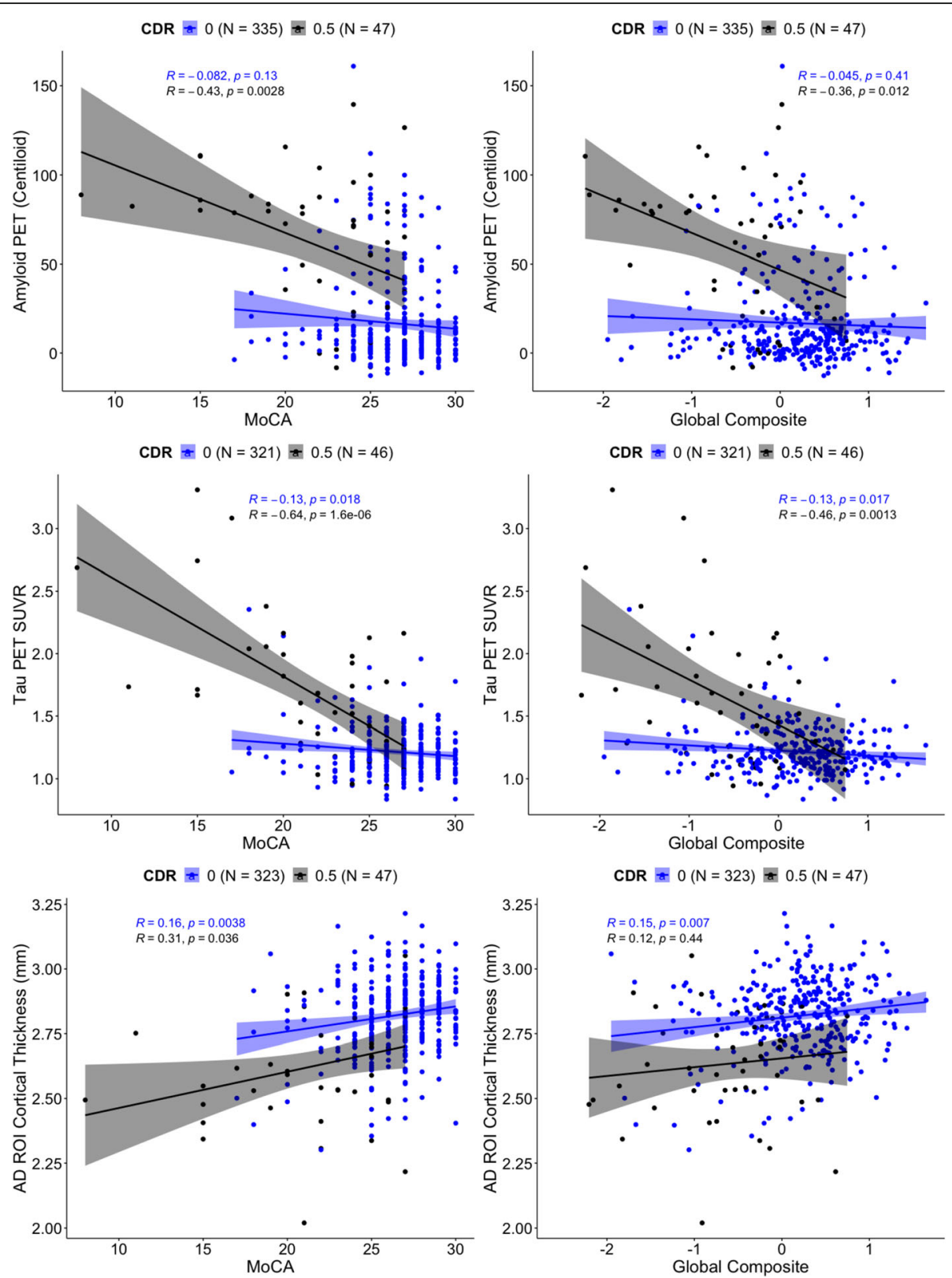

Fig. 2 Biomarker correlations with MoCA/UDS 3 global composite by CDR group

Knight ADRC sample did not support this hypothesis. There were only minor associations between the MoCA and long-form global composite measures and tau PET SUVR and cortical thickness in CDR 0 individuals. As expected, and consistent with prior work in this cohort $[41,42]$, more robust relationships were seen in CDR $0.5 \mathrm{~s}$ and significant effects extended to all three imaging markers, including amyloid PET. Although it may be somewhat surprising that amyloid PET was not significantly associated with either cognitive measure in the CDR 0 participants, this has been observed in previous studies. Specifically, relationships with amyloid and cognition are relatively inconsistent [43-45], and accumulating evidence suggests that changes in cognition in the preclinical stages of $\mathrm{AD}$ are predominantly driven by tau pathology and structural changes, rather than by amyloid alone $[41,46,47]$.

\section{Limitations}

The findings of this study should be considered in light of a number of limitations, which may be addressed in future studies. First, although it would be optimal to directly compare the performance of the short-form and 
long-form measures to one another in a single multiple regression, the high degree of correlation between the measures would result in potential multicollinearity issues if included in a single model together. Thus, our analyses were restricted to non-nested model comparison techniques. Second, we only compare baseline performance of the short-form and long-form measures here; it is possible that the long-form battery is more sensitive to decline than the short-form MoCA when assessing longitudinal change. Third, only a limited number of demographic covariates were included in the models presented. It is, of course, possible that the results may differ if other covariates were included. Finally, the participants included in the NACC cohort and the Knight ADRC cohort are composed of mostly highly motivated older adults who are comprehensively phenotyped and often engaged in imaging and fluid biomarker studies and therefore are not representative of the general population. Although a primary goal of the NIA ADRC programs and the Knight ADRC is to diversify enrollment to include underrepresented persons in aging and dementia research, the participants in these studies are primarily White and highly educated (see Tables 1 and 5).

\section{Conclusions}

Ultimately, the MoCA and UDS 3 global composite exhibit relatively equivalent, but limited, sensitivity to symptomatic conversion. Thus, depending on the use case, choosing the MoCA over longer cognitive batteries may afford sufficient sensitivity while also reducing administration time, costs, and participant burden. On the other hand, longer and more comprehensive measures do provide some unique power in predicting disease progression, depending on the cutoff point used. A shortcoming of both approaches is that neither demonstrated acceptable classification accuracy nor robust biomarker relationships in cognitively normal participants. In order for researchers and clinicians to reliably detect $\mathrm{AD}$ pathology in preclinical individuals, novel assessment methodologies with increased sensitivity and reliability (such as ecological momentary assessment studies or paradigms which move away from the traditional "one-shot" approach [48]) are necessary.

\footnotetext{
Acknowledgements

The NACC database is funded by NIA/NIH Grant U24 AG072122. NACC data are contributed by the NIA-funded ADRCs: P30 AG019610 (PI Eric Reiman, MD), P30 AG013846 (PI Neil Kowall, MD), P50 AG008702 (PI Scott Small, MD), P50 AG025688 (PI Allan Levey, MD, PhD), P50 AG047266 (PI Todd Golde, MD, PhD), P30 AG010133 (PI Andrew Saykin, PsyD), P50 AG005146 (PI Marilyn Albert, PhD), P50 AG005134 (PI Bradley Hyman, MD, PhD), P50 AG016574 (PI Ronald Petersen, MD, PhD), P50 AG005138 (PI Mary Sano, PhD), P30 AG008051 (PI Thomas Wisniewski, MD), P30 AG013854 (PI Robert Vassar, PhD), P30 AG008017 (PI Jeffrey Kaye, MD), P30 AG010161 (PI David Bennett, MD), P50 AG047366 (PI Victor Henderson, MD, MS), P30 AG010129 (PI Charles DeCarli, MD), P50 AG016573 (PI Frank LaFerla, PhD), P50 AG005131 (PI James
}

Brewer, MD, PhD), P50 AG023501 (PI Bruce Miller, MD), P30 AG035982 (PI Russell Swerdlow, MD), P30 AG028383 (PI Linda Van Eldik, PhD), P30 AG053760 (PI Henry Paulson, MD, PhD), P30 AG010124 (PI John Trojanowski, MD, PhD), P50 AG005133 (PI Oscar Lopez, MD), P50 AG005142 (PI Helena Chui, MD), P30 AG012300 (PI Roger Rosenberg, MD), P30 AG049638 (PI Suzanne Craft, PhD), P50 AG005136 (PI Thomas Grabowski, MD), P50 AG033514 (PI Sanjay Asthana, MD, FRCP), P30 AG066444 (PI John C. Morris, MD), and P50 AG047270 (PI Stephen Strittmatter, MD, PhD).

\section{Authors' contributions}

$J H, M L R, J N$, and AA were responsible for the study concept and design and drafting of the manuscript. JH, JN, and AA completed all the statistical analyses with critical input from $C X . J H, T B$, and $J M$ acquired funding and were responsible for the data collection. All authors provided critical feedback on the analyses and manuscript. All authors read and approved the final manuscript.

\section{Funding}

Study 1: See Acknowledgements.

Study 2: This work was supported by NIA Grants P01AG03991, P01AG026276, K01AG053474, and P30AG066444.

\section{Availability of data and materials}

The datasets used and/or analyzed during the current study are available from the corresponding author upon reasonable request.

\section{Declarations}

Ethics approval and consent to participate

Participants provided written informed consent, and all study procedures were approved by the individual ADRCs and approved by Institutional Review Boards as required for participation in NACC.

Consent for publication

Not applicable.

\section{Competing interests}

Neither Dr. Morris nor his family owns stock or has equity interest (outside of mutual funds or other externally directed accounts) in any pharmaceutical or biotechnology company. The other authors declare that they have no competing interests.

\section{Author details}

${ }^{1}$ Charles F. and Joanne Knight Alzheimer Disease Research Center, Department of Neurology, Washington University School of Medicine, St. Louis, MO, USA. ${ }^{2}$ Department of Psychological \& Brain Sciences, Washington University in St. Louis, St. Louis, MO, USA. ${ }^{3}$ Department of Radiology, Washington University School of Medicine, St. Louis, MO, USA. ${ }^{4}$ Division of Biostatistics, Washington University School of Medicine, St. Louis, MO, USA.

Received: 8 July 2021 Accepted: 24 August 2021

Published online: 13 September 2021

\section{References}

1. Twamley EW, Ropacki SA, Bondi MW. Neuropsychological and neuroimaging changes in preclinical Alzheimer's disease. J Int Neuropsychol Soc. 2006;12(5):707-35. https://doi.org/10.1017/S1355617706060863.

2. Vos SJ, Xiong C, Visser PJ, Jasielec MS, Hassenstab J, Grant EA, et al. Preclinical Alzheimer's disease and its outcome: a longitudinal cohort study. Lancet Neurol. 2013;12(10):957-65. https://doi.org/10.1016/S1474-4422(13 170194-7.

3. Edmonds EC, Delano-Wood L, Galasko DR, Salmon DP, Bondi MW. Subtle cognitive decline and biomarker staging in preclinical Alzheimer's disease. J Alzheimers Dis. 2015;47(1):231-42. https://doi.org/10.3233/JAD-150128.

4. Jack CR Jr, Bennett DA, Blennow K, Carrillo MC, Dunn B, Haeberlein SB, et al. NIA-AA research framework: toward a biological definition of Alzheimer's disease. Alzheimers Dement. 2018;14(4):535-62. https://doi.org/10.1016/j.ja Iz.2018.02.018.

5. Sliwinski MJ, Smyth JM, Hofer SM, Stawski RS. Intraindividual coupling of daily stress and cognition. Psychol Aging. 2006;21(3):545-57. https://doi. org/10.1037/0882-7974.21.3.545. 
6. Bambrah V, Hsu CF, Toplak ME, Eastwood JD. Anticipated, experienced, and remembered subjective effort and discomfort on sustained attention versus working memory tasks. Conscious Cogn. 2019;75:102812. https://doi.org/1 0.1016/j.concog.2019.102812

7. McGuire JT, Botvinick MM. Prefrontal cortex, cognitive control, and the registration of decision costs. Proc Natl Acad Sci. 2010;107(17):7922-6. https://doi.org/10.1073/pnas.0910662107.

8. Calamia M, Markon K, Tranel D. The robust reliability of neuropsychological measures: meta-analyses of test-retest correlations. Clin Neuropsychol. 2013; 27(7):1077-105. https://doi.org/10.1080/13854046.2013.809795.

9. Folstein MF, Folstein SE, McHugh PR. "Mini-mental state." A practical method for grading the cognitive state of patients for the clinician. J Psychiatr Res. 1975;12(3):189-98. https://doi.org/10.1016/0022-3956(75)9002 6-6.

10. Mitchell AJ. A meta-analysis of the accuracy of the mini-mental state examination in the detection of dementia and mild cognitive impairment. J Psychiatr Res. 2009;43(4):411-31. https://doi.org/10.1016/j.jpsychires.2008.04. 014.

11. Arevalo-Rodriguez I, Smailagic N, i Figuls MR, Ciapponi A, Sanchez-Perez E, Giannakou A, et al. Mini-Mental State Examination (MMSE) for the detection of Alzheimer's disease and other dementias in people with mild cognitive impairment (MCl). Cochrane Database Syst Rev. 2015;3:1-68.

12. Lacy M, Kaemmerer T, Czipri S. Standardized mini-mental state examination scores and verbal memory performance at a memory center: implications for cognitive screening. Am J Alzheimers Dis Other Demen ${ }^{\oplus}$. 2015;30(2): $145-52$.

13. Spencer RJ, Wendell CR, Giggey PP, Katzel LI, Lefkowitz DM, Siegel EL, et al. Psychometric limitations of the mini-mental state examination among nondemented older adults: an evaluation of neurocognitive and magnetic resonance imaging correlates. Exp Aging Res. 2013;39(4):382-97. https://doi. org/10.1080/0361073X.2013.808109.

14. Trzepacz PT, Hochstetler H, Wang S, Walker B, Saykin AJ. Relationship between the Montreal Cognitive Assessment and Mini-mental State Examination for assessment of mild cognitive impairment in older adults. BMC Geriatr. 2015;15(1):1-9.

15. Newman JC, Feldman R. Copyright and open access at the bedside. N Engl J Med. 2011;365(26):2447-9. https://doi.org/10.1056/NEJMp1110652.

16. de Silva V, Hanwella R. Why are we copyrighting science? BMJ. 2010;341:c4738.

17. Weintraub S, Besser L, Dodge HH, Teylan M, Ferris S, Goldstein FC, et al. Version 3 of the Alzheimer Disease Centers' neuropsychological test battery in the Uniform Data Set (UDS). Alzheimer Dis Assoc Disord. 2018:32(1):10-7. https://doi.org/10.1097/WAD.0000000000000223.

18. Besser L, Kukull W, Knopman DS, Chui H, Galasko D, Weintraub S, et al. Version 3 of the National Alzheimer's Coordinating Center's Uniform Data Set. Alzheimer Dis Assoc Disord. 2018;32(4):351-8. https://doi.org/10.1097/ WAD.0000000000000279

19. Nasreddine ZS, Phillips NA, Bédirian V, Charbonneau S, Whitehead V, Collin I, et al. The Montreal Cognitive Assessment, MoCA: a brief screening tool for mild cognitive impairment. J Am Geriatr Soc. 2005;53(4):695-9. https://doi. org/10.1111/j.1532-5415.2005.53221.x.

20. Pinto TC, Machado L, Bulgacov TM, Rodrigues-Júnior AL, Costa ML, Ximenes $\mathrm{RC}$, et al. Is the Montreal Cognitive Assessment (MoCA) screening superior to the Mini-Mental State Examination (MMSE) in the detection of mild cognitive impairment (MCl) and Alzheimer's Disease (AD) in the elderly? Int Psychogeriatr. 2019;31(4):491-504. https://doi.org/10.1017/S10416102180013 70.

21. Eguchi A, Kimura N, Aso Y, Yabuuchi K, Ishibashi M, Hori D, et al. Relationship between the Japanese version of the Montreal Cognitive Assessment and PET imaging in subjects with mild cognitive impairment. Curr Alzheimer Res. 2019;16(9):852-60. https://doi.org/10.2174/156720501 6666190805155230.

22. Morris JC. The clinical dementia rating (CDR): current version and scoring rules. Neurology. 1993;41:1588-92.

23. Carson N, Leach L, Murphy KJ. A re-examination of Montreal Cognitive Assessment (MoCA) cutoff scores. Int J Geriatr Psychiatry. 2018;33(2):379-88. https://doi.org/10.1002/gps.4756.

24. Holtzer R, Goldin Y, Zimmerman M, Katz M, Buschke H, Lipton RB. Robust norms for selected neuropsychological tests in older adults. Arch Clin Neuropsychol. 2008;23(5):531-41. https://doi.org/10.1016/j.acn.2008.05.004.

25. Jak AJ, Bondi MW, Delano-Wood L, Wierenga C, Corey-Bloom J, Salmon DP, et al. Quantification of five neuropsychological approaches to defining mild cognitive impairment. Am J Geriatr Psychiatry. 2009;17(5):368-75. https://doi. org/10.1097/JGP.0b013e31819431d5.

26. Petersen RC, Smith GE, Waring SC, Ivnik RJ, Tangalos EG, Kokmen E. Mild cognitive impairment: clinical characterization and outcome. Arch Neurol. 1999;56(3):303-8. https://doi.org/10.1001/archneur.56.3.303.

27. Storandt M, Morris JC. Ascertainment bias in the clinical diagnosis of Alzheimer disease. Arch Neurol. 2010;67(11):1364-9. https://doi.org/10.1 001/archneurol.2010.272.

28. Winblad B, Palmer K, Kivipelto M, Jelic V, Fratiglioni L, Wahlund LO, et al. Mild cognitive impairment-beyond controversies, towards a consensus: report of the International Working Group on Mild Cognitive Impairment. J Intern Med. 2004;256(3):240-6. https://doi.org/10.1111/j.1365-2796.2004.01380.x.

29. Therneau TM, Lumley T. Package 'survival'. R Top Doc. 2015;128(10):28-33.

30. Robin X, Turck N, Hainard A, Tiberti N, Lisacek F, Sanchez JC, et al. pROC: an open-source package for $\mathrm{R}$ and $\mathrm{S}+$ to analyze and compare ROC curves. BMC Bioinformatics. 2011;12(1):1-8.

31. Swets JA. Signal detection theory and ROC analysis in psychology and diagnostics: collected papers. New York: Psychology Press; 2014. https://doi. org/10.4324/9781315806167.

32. Su Y, Flores S, Hornbeck RC, Speidel B, Vlassenko AG, Gordon BA, et al. Utilizing the Centiloid scale in cross-sectional and longitudinal PiB PET studies. Neurolmage Clin. 2018;19:406-16. https://doi.org/10.1016/j.nicl.2018. 04.022

33. Su Y, Flores S, Wang G, Hornbeck RC, Speidel B, Joseph-Mathurin N, et al. Comparison of Pittsburgh compound B and florbetapir in cross-sectional and longitudinal studies. Alzheimers Dement Diagn Asses Dis Monit. 2019; 11(1):180-90.

34. Su Y, D'Angelo GM, Vlassenko AG, Zhou G, Snyder AZ, Marcus DS, et al. Quantitative analysis of PiB-PET with freesurfer ROIs. PLoS One. 2013;8(11): e73377. https://doi.org/10.1371/journal.pone.0073377.

35. Mishra S, Gordon BA, Su Y, Christensen J, Friedrichsen K, Jackson K, et al. AV1451 PET imaging of tau pathology in preclinical Alzheimer disease: defining a summary measure. Neuroimage. 2017;161:171-8. https://doi.org/1 0.1016/j.neuroimage.2017.07.050.

36. Head D, Rodrigue KM, Kennedy KM, Raz N. Neuroanatomical and cognitive mediators of age-related differences in episodic memory. Neuropsychology. 2008;22(4):491-507. https://doi.org/10.1037/0894-4105.22.4.491.

37. Raz N, Lindenberger U, Ghisletta P, Rodrigue KM, Kennedy KM, Acker JD. Neuroanatomical correlates of fluid intelligence in healthy adults and persons with vascular risk factors. Cereb Cortex. 2008;18(3):718-26. https:// doi.org/10.1093/cercor/bhm108.

38. Singh V, Chertkow H, Lerch JP, Evans AC, Dorr AE, Kabani NJ. Spatial patterns of cortical thinning in mild cognitive impairment and Alzheimer's disease. Brain. 2006;129(11):2885-93. https://doi.org/10.1093/ brain/awl256.

39. Fischl B, Dale AM. Measuring the thickness of the human cerebral cortex from magnetic resonance images. Proc Natl Acad Sci. 2000;97(20):11050-5. https://doi.org/10.1073/pnas.200033797.

40. Hassenstab J, Monsell SE, Mock C, Roe CM, Cairns NJ, Morris JC, et al. Neuropsychological markers of cognitive decline in persons with Alzheimer disease neuropathology. J Neuropathol Exp Neurol. 2015;74(11):1086-92. https://doi.org/10.1097/NEN.0000000000000254.

41. Aschenbrenner AJ, Gordon BA, Benzinger TL, Morris JC, Hassenstab J. Influence of tau PET, amyloid PET, and hippocampal volume on cognition in Alzheimer disease. Neurology. 2018;91(9):e859-66. https://doi.org/1 0.1212/WNL.0000000000006075.

42. Brier MR, Gordon B, Friedrichsen K, McCarthy J, Stern A, Christensen J, et al. Tau and A $\beta$ imaging, CSF measures, and cognition in Alzheimer's disease. Sci Transl Med. 2016;8(338):338ra66.

43. Dang C, Harrington KD, Lim YY, Ames D, Hassenstab J, Laws SM, et al. Relationship between amyloid- $\beta$ positivity and progression to mild cognitive impairment or dementia over 8 years in cognitively normal older adults. J Alzheimers Dis. 2018;65(4):1313-25. https://doi.org/1 $0.3233 / J A D-180507$

44. Baker JE, Lim YY, Pietrzak RH, Hassenstab J, Snyder PJ, Masters CL, et al. Cognitive impairment and decline in cognitively normal older adults with high amyloid- $\beta$ : a meta-analysis. Alzheimers Dement Diagn Asses Dis Monit. 2017:6:108-21.

45. Han SD, Nguyen CP, Stricker NH, Nation DA. Detectable neuropsychological differences in early preclinical Alzheimer's disease: a meta-analysis. Neuropsychol Rev. 2017;27(4):305-25. 
46. Mattsson-Carlgren N, Leuzy A, Janelidze S, Palmqvist S, Stomrud E, Strandberg $\mathrm{O}$, et al. The implications of different approaches to define AT (N) in Alzheimer disease. Neurology. 2020;94(21):e2233-44. https://doi.org/1 0.1212 WNL.00000000000009485.

47. Hanseeuw BJ, Betensky RA, Jacobs HI, Schultz AP, Sepulcre J, Becker JA, et al. Association of amyloid and tau with cognition in preclinical Alzheimer disease: a longitudinal study. JAMA Neurol. 2019;76(8):915-24. https://doi. org/10.1001/jamaneurol.2019.1424.

48. Hassenstab J, Aschenbrenner AJ, Balota DA, McDade E, Lim YY, Fagan AM, et al. Remote cognitive assessment approaches in the Dominantly Inherited Alzheimer Network (DIAN) Using digital technology to drive clinical innovation in brain-behavior relationships: a new era in neuropsychology. Alzheimers Dement. 2020;16:e038144.

\section{Publisher's Note}

Springer Nature remains neutral with regard to jurisdictional claims in published maps and institutional affiliations.

Ready to submit your research? Choose BMC and benefit from:

- fast, convenient online submission

- thorough peer review by experienced researchers in your field

- rapid publication on acceptance

- support for research data, including large and complex data types

- gold Open Access which fosters wider collaboration and increased citations

- maximum visibility for your research: over $100 \mathrm{M}$ website views per year

At BMC, research is always in progress.

Learn more biomedcentral.com/submissions 